\title{
Period Changes in the SX Phoenicis Star \\ CY Aquarii
}

\author{
Michael D. Joner and John M. Powell \\ Brigham Young University, Provo, Utah, U.S.A.
}

\begin{abstract}
CY Aquarii is a Population II dwarf Cepheid that has been observed on a regular basis since 1930 . We present evidence from unpublished times of maximum light from Brigham Young University observations secured in the mid-1970s to the present that indicate that the period of CY Aqr has changed in a discontinuous manner. Furthermore, we find that the period has changed significantly since the epoch of the last published investigation (1988). Finally, contrary to some past claims, examination of our high time resolution light curves yields no indication of multi-periodic pulsation in CY Aqr.
\end{abstract}

\section{Data Interpretation}

We utilize 84 unpublished times of maximum light secured between 1974 and 1991 at Brigham Young University along with about 500 light maxima from various literature sources to produce the O-C diagram for CY Aqr. Rolland et al. (1986) had suggested that the period for CY Aqr was changing in a continuous manner. In agreement with Percy (1975) and Kamper (1985), our observations indicate that the period has changed in a discontinuous manner over the years. The results of our period analysis are essentially indistinguishable from those of Coates et al. (1991) except for the fact that we find another sudden period change around 1989.4. The data were fit with a period of 0.061038302 days between 1968 and 1988 . The new period is slightly longer at 0.061038663 days. The latest period change is solid evidence that the period cannot be changing in a continuous manner since both of the previous changes had resulted in a slightly shorter period. The last of the Coates et al. (1991) observations were gathered in 1988. Analysis of differential BV observations indicate that any multi-periodic pulsation for CY Aqr must currently be of an amplitude that is less than or comparable to the noise $(<0.01 \mathrm{mag})$ in the photometry.

\section{References:}

Coates, D.W., Barnes, T.G., Fernley, J.A., Frueh, M.L. \& Sekiguchi, K., 1991, Delta Scuti Star Newsletter 4, 10

Kamper, B., 1985, IBVS, 2802

Percy, J.R., 1975, Astron. Astrophys. 43, 469

Rolland, A., Peña, J.H., Lopez de Coca, Peniche, R. \& Gonzalez, S.F., 1986, Astron. Astrophys. 168, 125 